\title{
CONTRACTION OPERATORS QUASISIMILAR TO A UNILATERAL SHIFT ${ }^{1}$
}

BY

\author{
V. T. ALEXANDER
}

\begin{abstract}
Let $U_{n}$ denote the unilaterial shift of finite multiplicity $n$. It is shown that a contraction operator $T$ is quasisimilar to $U_{n}$ if and only if $T$ is of Class $C_{1}$. , the canonical isometry $V$ associated with $T$ is pure and $T$ is $n$-cyclic with analytically independent vectors. For this, the notions of operators of analytic type and analytic independence of vectors are introduced. A characterization of the cyclic vectors of the Backward Shift is also presented.
\end{abstract}

0. Introduction. In this paper, necessary and sufficient conditions are obtained for a contraction operator to be quasisimilar to a unilateral shift. Two Hilbert space operators $T$ and $S$ are quasisimilar if there exist operators $X$ and $Y$ which are one-to-one, have dense range and satisfy $X T=S X$ and $T Y=Y S$. Quasisimilarity was introduced by Sz.-Nagy and Foiaş and they gave a simple characterization of operators quasisimilar to a unitary operator [7]. The topic was further studied by W. S. Clary who obtained a characterization of cyclic subnormal operators quasisimilar to the unilateral shift of multiplicity one [1]. W. W. Hastings generalized the result to the case of quasisimilarity between subnormal operators and isometries [5]. Both authors develop standard representations of the subnormal operators in terms of measures supported by $\bar{D}=\{z:|z| \leqslant 1\}$. Thus these subnormal operators are contractions and so an appropriate general question would be what type of contractions are quasisimilar to $U_{n}$ ?

1. Notation and definitions. A Hilbert space operator is a bounded linear transformation $T: H \rightarrow K$ from a Hilbert space $H$ into a Hilbert space $K$. If $K=H$, we say that $T$ is an operator on $H$. $T$ is said to be a contraction if $\|T\| \leqslant 1$. The set of analytic polynomials in one complex variable $z$ is denoted by $P$. Let $T$ be an operator on $H$. A subspace $M$ of $H$ is said to be invariant for $T$ if $T M \subseteq M$. For any given $f \in H$ let $P(f ; T)=\{p(T) f: p \in P\}$ and $M(f ; T)=$ closure of $P(f ; T)$ in $H$. Then $M(f ; T)$ is invariant for $T$. If there is a vector $f$ in $H$ such that $M(f ; T)=H$, we say that $T$ is cyclic and $f$ is a cyclic vector for $T$. For $n$ vectors $f_{1}, \ldots, f_{n}$ in $H$ let $M\left(f_{1}, \ldots, f_{n} ; T\right)$ be the closure of $P\left(f_{1}, \ldots, f_{n} ; T\right)=\left\{\sum_{i=1}^{n} p_{i}(T) f_{i}: p_{i} \in P\right\}$ in $H$. Then $M\left(f_{1}, \ldots, f_{n} ; T\right)$ is invariant for $T$. $T$ is said to be $n$-cyclic if there are $n$ vectors

Received by the editors September 24, 1982 and, in revised form, June 27, 1983.

1980 Mathematics Subject Classification. Primary 47B99.

Key words and phrases. Contraction, unilateral shift, operator of analytic type, isometry, quasisimilarity.

${ }^{1}$ This paper is adapted from the author's doctoral dissertation submitted at Fordham University. The author gratefully acknowledges his indebtedness to Professor William Hastings. 
$f_{1}, \ldots, f_{n}$ in $H$ with $M\left(f_{1}, \ldots, f_{n} ; T\right)=H$ while no smaller set of vectors has this property.

Let $T$ on $H$ and $S$ on $K$ be operators. An operator $X: H \rightarrow K$ is called a quasiaffinity if $X$ is one-to-one and has dense range. $T$ is said to be a quasiaffine transform of $S$ if there is a quasiaffinity $X: H \rightarrow K$ satisfying $X T=S X$. T and $S$ are said to be quasisimilar if each of them is a quasiaffine transform of the other. That is, if there exists quasiaffinities $X: H \rightarrow K$ and $Y: K \rightarrow H$ satisfying $X T=S X$ and $Y S=T Y$. It is known that the order of cyclicity is preserved under quasisimilarity. $T$ is said to be of class $C_{1}$. if $T^{n} h \nrightarrow 0$ for all $h$ in $H$ except $h=0$; of class $C_{10}$ if $T$ is of class $C_{1}$. and $T^{* n} h \rightarrow 0$ for all $h$ in $H$.

Let $T$ be a contraction on $H$. If $A$ is the algebra of functions analytic in $D=\{z$ : $|z|<1\}$ and continuous on $\bar{D}$ and $f \in A$ with power series $\sum a_{n} z^{n}$, then the operator series $\Sigma a_{n} T^{n}$ is convergent in norm to a limit denoted by $f(T)$. For $f \in H^{\infty}$ and $0<r<1$, the function $f_{r}$ defined by $f_{r}(z)=f(r z)$ belongs to $A$ and so $f_{r}(T)$ is an operator on $H$. Let $H_{T}^{\infty}$ denote the set of all functions $f$ in $H^{\infty}$ for which $f_{r}(T)$ has a strong limit as $r \rightarrow 1$ [7]. This limit is denoted by $f(T)$.

Definition 1.1. An operator $T$ on a space $H$ is said to be of analytic type if and only if $T$ is a contraction and $H_{T}^{\infty}=H^{\infty}$.

A completely nonunitary (c.n.u.) contraction is of analytic type [7, p. 111]. If $T$ is unitary and the spectral measure of $T$ is absolutely continuous with respect to $m$, normalized Lebesgue measure on the unit circle $C$, then $T$ is of analytic type [7, p. 116]. In particular, the bilateral shifts and the unilateral shifts are of analytic type. In general, a contraction $T$ is of analytic type if and only if the spectral measure of its unitary part is absolutely continuous.

Definition 1.2. Let $T$ be an operator of analytic type on the space $H$. Then $n$ vectors $f_{1}, \ldots, f_{n}$ in $H$ are said to be analytically independent under $T$ if a relation $F_{1}(T) f_{1}+\cdots+F_{n}(T) f_{n}=0$ with $F_{1}, \ldots, F_{n}$ in $H^{\infty}$ implies that $F_{1}=\cdots=F_{n}=0$.

An interesting result based on this notion of analytic independence is a simple characterization of the cyclic vectors of the backward shift $U^{*}$ given below. Here $U$ denotes the simple unilateral shift. The simple bilateral shift is denoted by $W$. The orthogonality of two vectors $f$ and $g$ is indicated by $f \perp g$. In the special case of $U$ or $W$ and for $F$ in $H^{\infty}$, the operator $F(U)$ or $F(W)$ is just multiplication by the function $F$ and so we will write $F f$ instead of $F(U) f$ or $F(W) f$. We will also use the fact that any function in the classical spaces $H^{p}, p>0$, is a quotient of two functions in $H^{\infty}$ [4].

THEOREM 1.3. A nonzero function in $H^{2}$ is a cyclic vector of $U^{*}$ if and only if 1 and $\bar{h}$ are analytically independent under $W(\bar{h}$ is the complex conjugate of $h)$.

Proof. Suppose $h$ is not a cyclic vector. Then there is a nonzero function $f$ in $H^{2}$ such that $f \perp U^{* n} h$ for all $n \geqslant 0$. That is, for $n=0,1,2, \ldots,\left(f, U^{* n} h\right)=\int z^{n} f \bar{h} d m$ $=0$. Since $f \bar{h}$ is in $L^{1}(m)$ it follows that $f \bar{h}$ is in $H^{1}$ and so is a quotient of $H^{\infty}$ functions, say, $f \bar{h}=G_{1} / G_{2}$. Also, $f$ being in $H^{2}$ is a quotient of $H^{\infty}$ functions: $f=F_{1} / F_{2}$. Thus $\left(F_{1} / F_{2}\right) \bar{h}=G_{1} / G_{2}$, or equivalently, $G_{2} F_{1} \bar{h}=F_{2} G_{1}$, showing that 1 and $\bar{h}$ are analytically dependent. 
Conversely, suppose $F \bar{h}=G$, where $F, G$ are nonzero functions in $H^{\infty}$. Since $G$ is in $H^{\infty}$ we have for $n=1,2, \ldots$,

$$
0=\int z^{n} G d m=\int z^{n} F \bar{h} d m=\left(U^{n} F, h\right)=\left(z F, U^{* n-1} h\right) .
$$

This shows that $z F$ is orthogonal to $U^{* k} h$ for $k=0,1,2, \ldots$ Since $z F$ is nonzero we conclude that $h$ is not a cyclic vector.

REMARKS. Several characterizations of the cyclic vectors of $U^{*}$ appear in [3]. Some of the other results in [3] can be obtained easily from the concept of analytic independence. The theorem also shows that polynomials, rational functions or inner functions cannot be cyclic vectors of $U^{*}$.

2. Quasisimilarity. For a contraction $T$ on $H$, we recall two constructions from [7]. First, $T$ has a canonical decomposition $T=T_{0} \oplus T_{1}$ on $H=H_{0} \oplus H_{1}$ such that $T_{0}$ on $H_{0}$ is unitary (called the unitary part) and $T_{1}$ on $H_{1}$ is completely nonunitary (called the c.n.u. part). Second, $T$ has a unitary dilation $U_{T}$ on a space $K$ having the following properties:

(1) $H$ is a subspace of $K$

(2) $T^{n} h=\operatorname{Pr} U_{T}^{n} h, n \geqslant 1, h \in H$,

where $\operatorname{Pr}$ denotes the orthogonal projection of $K$ onto $H$. $U_{T}$ is called minimal if the smallest subspace of $K$ which reduces $U_{T}$ and contains $H$ is all of $K$. If $T$ is c.n.u. then both $T$ and $U_{T}$ are of analytic type.

LEMMA 2.1. Let $T: H \rightarrow H$ be a contraction of analytic type. For every outer function $F$ in $H^{\infty}, F(T)$ is a quasiaffinity on $H$.

Proof. By the canonical decomposition of $T$ one reduces the lemma to Proposition 3.1 in [7] for the c.n.u. part and to the Riesz brothers' theorem for the unitary part.

Proposition 2.2. Let $T: H \rightarrow H$ be a contraction of analytic type. For all $n \geqslant 1$ the following are equivalent:

(a) There exists $n$ vectors $f_{1}, \ldots, f_{n}$ in $H$ which are analytically independent under $T$ and satisfying $M\left(f_{1}, \ldots, f_{n} ; T\right)=H$.

(b) There exists a quasiaffinity $Y: H_{n}^{2} \rightarrow H$ such that $Y U_{n}=T Y$.

Proof. Here $H_{n}^{2}$ denotes the direct sum of $n$ copies of $H^{2}$ on which $U_{n}$ acts in the usual manner. Let $e_{i}=(0, \ldots, 0,1,0, \ldots, 0)$ where the 1 occurs in the $i$ th place, $i=1, \ldots, n$.

(b) $\Rightarrow$ (a). Let $f_{i}=Y e_{i}$. Since $\left\{e_{i}: i=1, \ldots, n\right\}$ generate $H_{n}^{2}$ under $U_{n}$ it follows from the properties of $Y$ that $H=M\left(f_{1}, \ldots, f_{n} ; T\right)$. We assert that the $f_{i}$ are analytically independent under $T$. Let us assume the contrary, so that there are $H^{\infty}$ functions $F_{i}$, not all zero, satisfying $\Sigma F_{i}(T) f_{i}=0$. That is, $0=\Sigma F_{i}(T) Y e_{i}=Y\left(\Sigma F_{i} e_{i}\right)$. Since $Y$ is one-to-one, we must have $\Sigma F_{i} e_{i}=0$. This means $F_{i}=0$ for all $i$, which is a contradiction.

(a) $\Rightarrow$ (b). Let $U_{T}$ be the minimal unitary dilation of $T$ and $\mu_{i}$ be the measue corresponding to $f_{i}$ arising from the spectral measure of $U_{T}$. By hypothesis, $\mu_{i}$ is 
absolutely continuous with respect to $m$. Let $h_{i}=d \mu_{i} / d m$, so that $h_{i} \in L^{1}(m)$. Choose outer functions $F_{i}$ in $H^{\infty}$ satisfying $\left|F_{i}\right|^{2}=\left(1+h_{i}\right)^{-1}$ a.e. $(m)$ [6, p. 53].

Now we define $Y: H_{n}^{2} \rightarrow H$ by $Y e_{i}=F_{i}(T) f_{i}$ and extend to the set of vectors of the form $\left(p_{1}, \ldots, p_{n}\right)$, where $p_{i}$ are polynomials, by $Y\left(p_{1}, \ldots, p_{n}\right)=\Sigma p_{i}(T) F_{i}(T) f_{i}$. So $Y$ satisfies $Y U_{n}=T Y$. For polynomials $p_{1}, \ldots, p_{n}$,

$$
\begin{aligned}
\left\|Y\left(p_{1}, \ldots, p_{n}\right)\right\|_{H}^{2} & =\left\|\sum p_{i}(T) F_{i}(T) f_{i}\right\|^{2} \leqslant n \cdot \sum\left\|p_{i}(T) F_{i}(T) f_{i}\right\|^{2} \\
& \leqslant n \cdot \sum\left\|p_{i}\left(U_{T}\right) F_{i}\left(U_{T}\right) f_{i}\right\|^{2}=n \cdot \sum \int\left|p_{i} F_{i}\right|^{2} d \mu_{i} \\
& =n \cdot \sum \int\left|p_{i}\right|^{2} h_{i}\left(1+h_{i}\right)^{-1} d m \\
& \leqslant n \cdot \sum\left\|p_{i}\right\|_{H^{2}}^{2}=n \cdot\left\|\left(p_{1}, \ldots, p_{n}\right)\right\|_{H_{n}^{2}}^{2} .
\end{aligned}
$$

Thus $Y$ is bounded on the set of vectors of the form $\left(p_{1}, \ldots, p_{n}\right)$. Since this set is dense in $H_{n}^{2}, Y$ has a unique bounded extension to all of $H_{n}^{2}$.

To show that $Y$ is one-to-one, suppose that $Y\left(g_{1}, \ldots, g_{n}\right)=0$. Each $g_{i}$ is in $H^{2}$ and so is a quotient of two $H^{\infty}$ functions: $g_{i}=G_{1 i} / G_{2 i}$, where $G_{2 i}$ are outer. Let $G_{2}=\prod_{i=1}^{n} G_{2 i}$ and $G_{2 i}^{\prime}=G_{2} / G_{2 i}$. Then we have

$$
\begin{aligned}
0 & =Y\left(g_{1}, \ldots, g_{n}\right)=G_{2}(T) Y\left(g_{1}, \ldots, g_{n}\right)=Y\left(G_{2}\left(g_{1}, \ldots, g_{n}\right)\right) \\
& =Y\left(G_{21}^{\prime} G_{11}, \ldots, G_{2 n}^{\prime} G_{1 n}\right)=Y\left(\sum G_{2 i}^{\prime} G_{1 i} e_{i}\right)=\sum\left(G_{2 i}^{\prime} G_{1 i}\right)(T) F_{i}(T) f_{i} .
\end{aligned}
$$

Since $G_{2 i}^{\prime}, F_{i}$ are outer functions, the analytic independence of $f_{i}$ under $T$ implies that $G_{1 i}=0$ for all $i$. That is, $g_{i}=0$ for all $i$.

Next, we show that $Y$ has dense range. Suppose $p$ is a polynomial. We have

$$
\int\left|p / F_{i}\right|^{2} d m=\int|p|^{2}\left(1+h_{i}\right) d m<\infty .
$$

Hence by [4, Theorem 2.11], $p / F_{i}$ is in $H^{2}$. Write $g_{i}=p / F_{i}$. Then $g_{i}$ is in $H^{2}$ and $F_{i} g_{i}=p$. Then

$$
\begin{aligned}
F_{i}(T) Y\left(g_{i} e_{i}\right) & =Y\left(F_{i} g_{i} e_{i}\right)=Y\left(p e_{i}\right)=p(T) Y e_{i} \\
& =p(T) F_{i}(T) f_{i}=F_{i}(T) p(T) f_{i} .
\end{aligned}
$$

Since $F_{i}$ is outer, from Lemma 2.1 we get $Y\left(g_{i} e_{i}\right)=p(T) f_{i}$. It is now clear that $\operatorname{ran} Y$ contains all vectors of the form $\Sigma p_{i}(T) f_{i}$, where $p_{i}$ are polynomials. Thus $Y$ has dense range and the proof is complete.

We recall the following results from [7].

PROPOSITION 2.3 (Wold DECOMPOSITION [7, p. 3]). Every isometry is the direct sum of a unitary operator and a unilateral shift.

If the isometry has no unitary part it is said to be pure. Thus a pure isometry is a unilateral shift.

Proposition 2.4. Every isometry has a minimal unitary extension $[7, p .6]$.

The minimal unitary extension of $U_{n}$ is $W_{n}$, the bilateral shift of multiplicity $n[7$, p. 5]. 
Proposition 2.5. Suppose $T$ is a contraction of class $C_{1}$. on the space $H$. There is a positive quasiaffinity $X$ on $H$ of norm one and an isometry $V$ on $H$ such that $X T=V X$. Further, for all $h$ in $H,\|X h\|=\inf _{k}\left\|T^{k} h\right\|[7$, p. 79].

We shall call this isometry $V$ the canonical isometry associated with $T$. If $T$ has a unitary part then it is easy to see that $V$ has a unitary part. Therefore $V$ pure implies that $T$ is c.n.u.

Proposition 2.6. Let $F_{i}=\left(f_{i 1}, \ldots, f_{i n}\right), i=1, \ldots, n$, be any $n$ vectors in $H_{n}^{2}$. Then $F_{i}$ are analytically dependent if and only if $\operatorname{det}\left(f_{i j}\right)=0$.

Proof. Let $A$ denote the matrix $\left(f_{i j}\right)$. Since $f_{i j}$ are in $H^{2}$, det $A$ is a function of class $N^{+}$. Assume that $\operatorname{det} A=0$. Hence for all $z$ in $D$ we have $\operatorname{det} A(z)=$ $\operatorname{det}\left(f_{i j}(z)\right)=0$. Let $k(z)$ be the row rank of $A(z)$ and put $k=\max _{z \in D} k(z)$. Then $0<k<n$. Thus there exists $z$ in $D$ and a $k \times k$ minor of $A(z)$ whose determinant is not zero, while every minor of $A(z)$ of order greater than $k$ has determinant zero for all $z$ in $D$. Without loss of generality, we may assume that the principal $k \times k$ minor $B=\left(f_{i j}\right), 1 \leqslant i, j \leqslant k$, has the nonzero determinant at some point $z$ in $D$. Thus $\operatorname{det} B$ is a nonzero function of class $N^{+}$. Let

$$
\left(h_{1}, \ldots, h_{k}\right)=\left(f_{k+1,1}, \ldots, f_{k+1, k}\right) \cdot \operatorname{det} B \cdot B^{-1} .
$$

Then each $h_{i}$ is of class $N^{+}$. Hence there exists a nonzero function $F$ in $H^{\infty}$ such that $F h_{1}, \ldots, F h_{k}$ and $F \cdot \operatorname{det} B$ are all $H^{\infty}$ functions. This follows from the fact that every function of class $N^{+}$is a quotient of two functions in $H^{\infty}$ [4]. We claim that

$$
(F \cdot \operatorname{det} B) \cdot F_{k+1}=F \cdot \sum_{i=1}^{k} h_{i} F_{i} \text {. }
$$

To see this, let us set $G_{i}=\left(f_{i 1}, \ldots, f_{i k}\right), i=1, \ldots, k+1$. Then by our choice of $h_{1}, \ldots, h_{k}$, we have $F \cdot \operatorname{det} B \cdot G_{k+1}=F \cdot \sum h_{i} G_{i}$. Hence, if $(*)$ were not true at some point $z_{0}$ in $D$, then $A\left(z_{0}\right)$ has a minor of order $k+1$ whose determinant is not zero, contradicting our choice of $k$. From $(*)$ we conclude that $F_{1}, \ldots, F_{k+1}$ and hence $F_{1}, \ldots, F_{n}$ are analytically dependent.

Conversely, suppose that $F_{1}, \ldots, F_{n}$ are analytically dependent: $\Sigma h_{i} F_{i}=0$, where $h_{i}$ are $H^{\infty}$ functions, not all zero. Thus for all $z$ in $D$ we have $\Sigma h_{i}(z) F_{i}(z)=0$. That is, for all $z$ in $D$,

$$
\left(h_{1}(z), \ldots, h_{n}(z)\right) \cdot\left(f_{i j}(z)\right)=0 .
$$

Hence $\operatorname{det}\left(f_{i j}(z)\right)=0$ for all points $z$ in $D$ at which at least one of the numbers $h_{1}(z), \ldots, h_{n}(z)$ is different from zero. But the common zeros of $h_{1}, \ldots, h_{n}$ are at most countable and so we must have $\operatorname{det}\left(f_{i j}\right)=0$.

COROLlaRY 2.7. Any $n+1$ vectors in $H_{n}^{2}$ are analytically dependent.

Proof. Let $F_{i}=\left(f_{i 1}, \ldots, f_{i n}\right), i=1, \ldots,(n+1)$, be $n+1$ vectors in $H_{n}^{2}$. We may assume that the first $n$ vectors $F_{1}, \ldots, F_{n}$ are analytically independent. By Proposition 2.6 we have

$$
\operatorname{det}\left(f_{i j}\right)_{1 \leqslant i, j \leqslant n} \neq 0 \text {. }
$$


Let $\left(h_{1}, \ldots, h_{n}\right)=F_{n+1} \cdot \operatorname{det}\left(f_{i j}\right) \cdot\left(f_{i j}\right)^{-1}$. Then there exists a function $F$ in $H^{\infty}$ such that $F h_{1}, \ldots, F h_{n}$ and $F \cdot \operatorname{det}\left(f_{i j}\right)$ are all in $H^{\infty}$. We obtain

$$
F \cdot \operatorname{det}\left(f_{i j}\right) \cdot F_{n+1}=F \cdot\left(h_{1}, \ldots, h_{n}\right) \cdot\left(f_{i j}\right)=\sum_{i=1}^{n} F h_{i} F_{i},
$$

showing that $F_{1}, \ldots, F_{n+1}$ are analytically dependent.

THEOREM 2.8. Let $T$ be a contraction on the space $H$. Then $T$ is quasisimilar to $U_{n}$ if and only if the following conditions hold:

(1) $T$ is of class $C_{1}$..

(2) The canonical isometry $V$ associated with $T$ is pure.

(3) There exists $n$ vectors $f_{1}, \ldots, f_{n}$ in $H$ which are analytically independent under $T$ and satisfying $M\left(f_{1}, \ldots, f_{n} ; T\right)=H$.

Proof. The conditions are sufficient. Condition (2) implies that $T$ is c.n.u. and so is of analytic type. By condition (3) and Proposition 2.2 there is a quasiaffinity $Y$ : $H_{n}^{2} \rightarrow H$ satisfying $Y U_{n}=T Y$. By condition (1) we have the quasiaffinity $X$ on $H$ satisfying $X T=V X$, where $V$ is the canonical isometry associated with $T$. From (2), $V$ is a unilateral shift. From $M\left(f_{1}, \ldots, f_{n} ; T\right)=H$ and $X T=V X$ it follows that $M\left(X f_{1}, \ldots, X f_{n} ; V\right)=H$. This shows that $V$ has multiplicity at most $n$.

Suppose that the multiplicity of $V$ is $k$ where $k<n$. Indentifying $V$ with $U_{k}$ and applying Corollary 2.7 we see that $X f_{1}, \ldots, X f_{n}$ are analytically dependent under $V$. Hence there exists $H^{\infty}$ functions $F_{1}, \ldots, F_{n}$, not all zero, satisfying $\Sigma F_{i}(V) X f_{i}=0$. This means $X\left(\Sigma F_{i}(T) f_{i}\right)=0$ and hence $\Sigma F_{i}(T) f_{i}=0$. This contradicts (3) and so $V$ must have multiplicity $n$.

The conditions are necessary. We are assuming that $T$ is quasisimilar to $U_{n}$. Let $Y$ : $H \rightarrow H_{n}^{2}$ and $Z: H_{n}^{2} \rightarrow H$ be quasiaffinities satisfying $Y T=U_{n} Y$ and $Z U_{n}=T Z$.

(1) $T$ is of class $C_{1}$. Let $h$ be any vector in $H$ such that $T^{k} h \rightarrow 0$. Then $Y T^{k} h \rightarrow 0$. This implies $U_{n}^{k} Y h \rightarrow 0$. But, clearly, $\left\|U_{n}^{k} Y h\right\|=\|Y h\|$ for all $k$ and so we have $h=0$.

Now $\left(U_{n}^{*}\right)^{k} \rightarrow 0$ strongly as $k \rightarrow \infty$ implies that $T^{* k} \rightarrow 0$ strongly. Thus $T$ is of class $C_{10}$ and so is c.n.u. and of analytic type.

(2) The canonical isometry $V$ is pure: By Proposition 2.5 we have the quasiaffinity $X$ satisfying $X T=V X$. From $Y: H \rightarrow H_{n}^{2}$ we construct an operator $Y_{0}: H \rightarrow H_{n}^{2}$ with the properties $Y_{0} V=U_{n} Y_{0}$ and $Y_{0} X=Y$ as follows.

Just define $Y_{0} X h=Y h$ for all $h$ in $H$. Then $Y_{0}$ is densely defined and has dense range. For $h$ in $H$ we have for $k=1,2, \ldots$,

$$
\left\|Y_{0} X h\right\|=\|Y h\|=\left\|U_{n}^{k} Y h\right\|=\left\|Y T^{k} h\right\| \leqslant\|Y\| \cdot\left\|T^{k} h\right\| .
$$

It follows that

$$
\left\|Y_{0} X h\right\| \leqslant\|Y\| \cdot \inf _{k}\left\|T^{k} h\right\|=\|Y\| \cdot\|X h\|,
$$

by Proposition 2.5. Thus $Y_{0}$ is bounded by $\|Y\|$ on the dense set of ran $X$. So it has a unique extension to all of $H$ satisfying $\left\|Y_{0}\right\| \leqslant\|Y\|$. Now

$$
Y_{0} V X h=Y_{0} X T h=Y T h=U_{n} Y h=U_{n} Y_{0} X h
$$


shows that $Y_{0} V$ and $U_{n} Y_{0}$ agree on the dense set ran $X$. Hence we must have $Y_{0} V=U_{n} Y_{0}$. The construction of $Y_{0}$ is complete.

Now $Y_{0} V=U_{n} Y_{0}$ implies that $V X Z Y_{0}=X Z Y_{0} V$. Let $V_{1}$ denote the minimal unitary extension of $V$. Since $V X Z=X Z U_{n}$ one infers that $V_{1}$ has finite multiplicity and so cannot be unitarily equivalent to any proper part of it. Now let $Y_{1}$ be the unique bounded lifting of $X Z Y_{0}$ commuting with $V_{1}$ [2, Corollary 5.1]; obviously $Y_{1}$ has dense range. By [2, Lemma 4.1], $V_{1}$ is unitarily equivalent to $V_{1} \mid\left(\operatorname{ker} Y_{1}\right)^{\perp}$ and it follows from $\operatorname{ker} Y_{0} \subset \operatorname{ker} Y_{1}=(0)$ that $Y_{0}$ is a quasiaffinity. Therefore, from $V^{* k} Y_{0}^{*}$ $=Y_{0}^{*} U_{n}^{* k} \rightarrow 0$ strongly as $k \rightarrow \infty$, we infer that $V^{* k} \rightarrow 0$ strongly as $k \rightarrow \infty$. This means that $V$ is pure.

(3) Take $f_{i}=Z e_{i}$. Then $f_{1}, \ldots f_{n}$ satisfy the requirements. For, $Z$ is a quasiaffinity implies $M\left(f_{1}, \ldots, f_{n} ; T\right)=H$. The analytic independence of $f_{1}, \ldots, f_{n}$ under $T$ also follows easily.

\section{REFERENCES}

I. W. S. Clary, Quasisimilarity and subnormal operators, Ph.D. thesis, The University of Michigan, 1973.

2. R. G. Douglas, On the operator equation $S^{*} X T=X$, Acta. Sci. Math. (Szeged) 30 (1969), 19-32.

3. R. G. Douglas, H. S. Shapiro and A. L. Shields, Cyclic vectors and invariant subspaces for the backw'ard shift operator, Ann. Inst. Fourier (Grenoble) 20 (1970), 37-76.

4. P. L. Duren, Theory of $H^{p}$ spaces, Academic Press, New York, 1970.

5. W. W. Hastings, Subnormal operators quasisimilar to an isometry, Trans. Amer. Math. Soc. 256 (1979), 145-161.

6. K. Hoffman, Banach spaces of analytic functions, Prentice-Hall, Englewood Cliffs, N.J., 1962.

7. B. Sz.-Nagy and C. Foiaş, Harmonic analysis of operators on Hilhert space, American Elsevier, New York, 1970.

Department of Mathematics, Medgar Evers College of The City University of New York, BROOKLYN, NEW YORK 11225 